\section{Simultaneous Transmission and Reception of Radio Waves}

WE have succeeded in operating a valve receiver $K_{1}$ and a valve transmitter $T_{1}$ both working on the same antenna, and during their operation, receiving through $R_{1}$ another distant transmitter $T_{2}$, working on exactly the same frequency as $T_{1}$. This has been done by a device making use of simultaneous modulation of $T_{1}$ and $R_{1}$.

The frequencies used are about 11 megacycles per sec. for the carrier waves of $T_{1}$ and $T_{2}$, and 750 cycles/sec. for modulation. We have also worked with lower frequencies, for example, a carrier wave of 1 megacycle/sec. and modulation frequencies of $50-16,000$ cycles/sec.

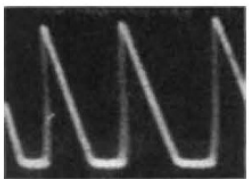

Fra. 1.

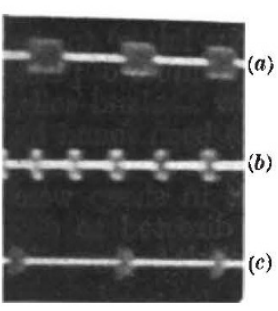

FIG. 2.
A special wave-form of modulation other than the common sinusoidal wave has been advantageously used for this purpose. This wave-form as well as the transmitted groups of waves can be observed during actual work, by a cathode ray oscillograph. Fig. 1 is from a photograph of such a wave. form used for modulation (frequency 750 cycles/sec.) Fig. $2 a$ shows a pattern of transmitted groups of waves of a frequency one megacycle/sec. modulated by 750 cycles/sec.; Fig. $2 b$ is the same pattern as Fig. $2 a$ modulated at double frequency, that is, 1,500 cycles/sec.; Fig. $2 c$ is the same pattern as Fig. $2 a$, but using for the transmitting valve a high negative grid-bias near to the point at which the modulated transmitter $T_{1}$ stops oscillation.

Owing to the development of short-wave technique, this problem of simultaneous transmission and reception may form the basis of many future scientific: and technical applications.

Henry Antoun. Faris Minaw.

Physics Department,

Egyptian University

Cairo.

Aug. 18.

\section{A Cosmic Cyclotron as a Cosmic Ray Generator?}

MaNY hypotheses have been propounded regarding the origin of cosmic radiation, but none of them seems to be able to account for the enormous energies found. In this note I shall try to show that a double star may constitute a gigantic cyclotron, which can give rays with energies of the right order of magnitude.

Let us assume that a charged particle (for example, an electron) moves in the magnetic equatorial plane of a star. If the field is $H$ (equal to a constant multi plied by $R^{-3}$ ), the particle moves in a small circle with radius $\rho$ which has a precessional movement around the star in a circle with radius $R$ (Fig. 1). The angular velocity of the precession is

$$
\omega=\frac{3}{2} v \frac{p}{R^{2}},
$$

where $v$ is the velocity of the particle. If $v$ is very near $c$, the velocity of light, we have $p=V / 300 H$, if $e V$ is the energy of the particle in electron volts. Then we get :

$$
\omega=\frac{c}{200 R^{2}} \quad \frac{V}{H}
$$

We now assume that a planet, or a second star, rotates around the first star, with the same angular velocity $\omega$. Then an electric field $F$ (deriving from a difference of potential between the stars or from the rotation) can constantly increase the energy of the particle in the same way as a rotating electric field accelerates ions in a cyclotron. When the energy. of the particle increases, $R$ is diminished and the particle seems to be in a rather stable equilibrium in relation to the rotating system.
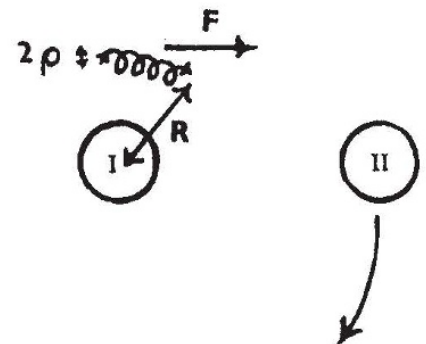

FIG. 1

The maximum energy of the particle can be calculated from (1). If $R$ is $7 \times 10^{10} \mathrm{~cm}$. (about the radius of our sun) and $H$ is 50 gauss (about the magnetic field at the surface of the sun), and further, $\omega=0.5 \times 10^{-4} \mathrm{sec}^{-1}$ (a reasonable value for the rotation of a double star), we get

$$
V \sim 10^{11} \text { electron volts }
$$

which is of the order of magnitude of the energy of the bursts.

Physics Laboratory,

Hannes Alfrvén.

University, Uppsala.

Oet. 4.

\section{Wilhelm von Waldeyer}

IN the note on Wilhelm von Waldeyer in Nature of October 3, p. 579, there appears the statement that this German anatomist was the first to describe the neurone theory. Most discoveries have been based on the roork of previous investigators, and there is much useless discussion on priority concerning almost every great contribution to pure or applied science. In the present instance, however, a survey of the relevant literature readily shows that Waldeyer's only significant contribution to the neurone theory with which his name is so often linked was the introduction of the term 'neurone'.

The doctrine that the nervous system is formed of cells is a corollary of the cellular theory of Schwann and Schleiden. Leeuwenhoek and Malpighi saw cells ('globuli') in the cerebral cortex and, so early as 1741, Swedenborg, in his "Oeconomia Regni Animalis", described the cortical cells and their supposed functional importance in a manner surprisingly similar to the views which are now held. Waller's work (1850) on the degeneration of nerve fibres showed. the trophic influence of the nerve cell on the fibre. Deiters, in 1865 , showed that each nerve cell possessed an axis cylinder and dendrites. His 